\title{
Es ist Zeit für eine neue Interdisziplinarität in der Wissenschaft!
}

DOI: $10.1007 / \mathrm{s} 12268-020-1380-8$ (C) Der Autor 2020

In der Corona-Pandemie haben wir die Bedeutung von Wissenschaft für moderne Gesellschaften eindrucksvoll erfahren dürfen. Selten hat Politik in solcher Intensität auf faktenbasierte Erkenntnisse und Ratschläge gehört wie in den letzten Wochen.

Gleichzeitig wurde deutlich, wie wichtig in diesem Zusammenhang interdisziplinäre Kooperation ist. Denn nach einer Phase des alleinigen Primates des Gesundheitsschutzes, ging es in der Krise bald um die Abwägung gesundheitswissenschaftlicher, ökonomischer und gesellschaftlicher Zielkonflikte. Hierfür mussten Wissenschaftlerinnen und Wissenschaftler sehr unterschiedlicher Disziplinen zusammenwirken.

Die Nationale Akademie der Wissenschaften Leopoldina als Zusammenschluss führender Wissenschaftler/innen unterschiedlichster Disziplinen hat mit ihren interdisziplinär getragenen Empfehlungen dabei Wichtiges geleistet.

Gleichzeitig wird deutlich, dass es im Wissenschaftssystem zu wenig Orte gibt, in denen eine solche Interdisziplinarität auch im Normalbetrieb kultiviert wird. Der Wissenschaftsbetrieb lebt von seiner methodischen Ausdifferenzierung in Disziplinen und Teildisziplinen.

Interdisziplinarität entfaltet sich zumeist an den Grenzen von unmittelbaren Nachbardisziplinen. Der Brückenschlag von den Natur- und Technikwissenschaften in die Wirtschafts-, Sozial- und Geisteswissenschaften ist eher noch selten. Zu unterschiedlich sind die Methoden und Wissenschaftskulturen in den beiden Welten, gerade dann, wenn auch die wissenschaftlich begründete Auseinandersetzung mit Wertentscheidungen notwendig ist: Denn während sich Naturwissenschaften auf das konzentrieren, was ist, setzen sich Gesellschafts- und Geisteswissenschaften auch mit der Frage auseinander, wie sich begründen lässt, was in Gesellschaften sein kann und soll.

Doch je mehr Wissenschaft auch künftig bei der Gestaltung von gesellschaftlichen Entscheidungen und Veränderungsprozessen als Kompassgeber gefragt ist, desto wichtiger wird es, dass diese erweiterten Formen des Brückenschlages immer öfter zum Regelfall werden. Naturwissenschaftlich-technische, ökonomische, politisch-institutionelle und gesellschaftlich-kulturelle Dimensionen des Wandels müssen wissenschaftlich zusammengedacht werden. Es braucht eine transformative literacy, die alle diese Dimensionen im Blick behält. Sie gibt Orientierung in komplexen Transformationsprozessen.

Jenseits der nationalen Wissenschaftsakademien braucht es deswegen mehr Orte und Anreize für diese erweiterte Form der Interdisziplinarität.

Dies erfordert Weiterentwicklungen des Wissenschaftssystems auf sehr unterschiedlichen Ebenen: Für Wissenschaftler/innen, die sich erweiterten Formen der interdisziplinären Kooperation öffnen, müssen Qualifizierungs- und Karrieremöglichkeiten offenstehen. Heute verlaufen wissenschaftliche Karrieren immer noch sehr stark orientiert an der jeweiligen wissenschaftlichen Teildisziplin: Dort sitzen die für die weitere Karriere entscheidenden Peers. Inter- oder sogar transdisziplinäre Journale haben oft geringere Impact-Faktoren als die rein fachwissenschaftlichen Spitzenjournale. Für wirklich interdisziplinäre Doktorarbeiten bauen die Promotionsordnungen vieler Fachbereiche oft hohe Hürden in der Zulassung und kompetenten Betreuung auf.

Die Weiterentwicklung dieser Anreiz- und Qualifizierungssysteme würde auch dadurch erleichtert, dass mehr Institutionen entstehen, die bei der Berufung von Spitzenpersonal auf solche breit interdisziplinär ausgerichtete Wissenschaftler/innen angewiesen sind. Für eine disziplinenübergreifende Gesellschaftsberatung sollten daher Zentren für interdisziplinäre Transformationsforschung initiiert werden. Sie wären Anlaufstelle für Politik und Gesellschaft auch in Normalzeiten, um Orientierung für politische Entscheidungen z. B. im Kontext von Pandemien oder dem Klimawandel aus multidisziplinärer Perspektive zu geben. Solche Zentren könnten an Universitäten, aber auch außeruniversitär entstehen. Sie könnten Initiator angesehener Fachjournale werden. Hier ließen sich Doktorandenschulen für interdisziplinäre Promotionen ansiedeln. So würden Orte für Wissenschaftler/innen geschaffen, die sich schon früh der Mühe interdisziplinärer Kooperation stellen und die entsprechenden Kompetenzen aufbauen.

Die Corona-Krise hat dem Wissenschaftssystem neue Legitimation und Rückenwind gegeben. Diese gilt es für eine kluge Weiterentwicklung interdisziplinärer Forschungsstrukturen zu nutzen.

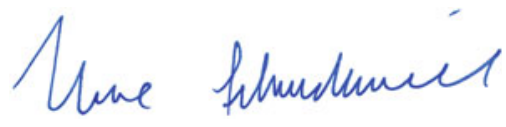

Prof. Dr. Uwe Schneidewind

Präsident des Wuppertal Institutes für Klima, Umwelt, Energie (2010-2020) sowie ehemaliger Präsident der Universität Oldenburg

\section{Funding: Open Access funding provided by Projekt DEAL. \\ Open Access: Dieser Artikel wird unter der Creative Commons Namensnennun 4.0 International Lizenz veröffentlicht, welche die Nutzung, Vervielfältigung, Bearbeitung, Verbreitung und Wiedergabe in jeglichem Medium und Form ordnungssemä nennen, einen Link zur Creative Comm die Quelle angeben, ob Änderungen vorgenommen wurden. Die in diesem Artike enthaltenen Bilder und sonstiges Drittmaterial unterliegen ebenfalls der genannten Creative Commons Lizenz, sofern sich aus der Abbildungslegende nichts anderes ergibt. Sofern das betreffende Material nicht unter der genannten Creative Commons Lizenz steht und die betreffende Handlung nicht nach gesetzlichen Vorschriften erlaubt ist, ist für die oben aufgeführten Weiterverwendungen des Materials die Einwilligung des jeweiligen Rechteinhabers einzuholen. Weitere Details zur Lizenz entnehmen Sie bitte der
Lizenzinformation auf http://creativecommons.org/licenses/by/4.0/deed.de.}

Korrespondenzadresse:

Prof. Dr. Uwe Schneidewind

Wuppertal Institut für Klima, Umwelt, Energie

Döppersberg 19

D-42103 Wuppertal

uwe.schneidewind@wupperinst.org

www.wupperinst.org 